\title{
Male display characters, gonadal maturation and androgens in the cichlid fish Oreochromis mossambicus
}

Received: 23 February 1999 / Received in revised form: 3 May 1999 / Accepted: 10 May 1999

\begin{abstract}
The relationships between gonadal maturation and the expression of behavioral and morphological display characters in $O$. mossambicus was investigated and the possible role of androgens in the coordination of this process was evaluated using genital papilla size as a bioassay for androgen levels. Morphological and behavioral displaying characters were found to be correlated among themselves. The sexually dimorphic characters were correlated with gonad maturation and androgen levels, but not with the gonado-somatic index. Gonad maturation and androgen levels were closely related. Territoriality and other behavioral variables involved in reproduction were also correlated with gonad maturation and androgen levels. These data suggest that in O. mossambicus androgens play a mediating role between gonadal maturation and the expression of display characters, both morphological and behavioral.
\end{abstract}

Key words Androgens · Cichlids · Maturation · Reproductive behaviour $\cdot$ Secondary sex characters

\section{Introduction}

From an evolutionary point of view, it is likely that the maturation of behavioral traits involved in mate acquisition and that of morphological sex-dimorphic traits are closely associated with gonadal maturation. This is because many of these behavioral and morphological traits (displaying characters) involve costs to the individuals possessing them, costs that are only compensated when these individuals are effectively capable of mating. There is a large body of evidence that demonstrates that secondary sex traits raise the risk of predation (Magnhagen 1991) and result in other constraints such as higher energetic costs (Halliday 1987).

R.F. Oliveira ( ) V.C. Almada

Unidade de Investigação em Eco-Etologia,

Instituto Superior de Psicologia Aplicada,

Rua Jardim do Tabaco 44, P-1100 Lisbon, Portugal

e-mail: ruiol@ispa.pt

Tel: +351-1-8811700, Fax: +351-1-8860954
Because gonadal androgens are secreted by cell populations intimately associated with sperm-producing cells (Fostier et al. 1983; Nagahama 1983), and modulate the expression of both behavioral and morphological traits (Borg 1994), they are especially suited as coordinating agents.

Oreochromis mossambicus (Peters) is an African mouth-brooding cichlid in which males form dense leklike aggregations that females visit when ready to spawn. Mating occurs inside a spawning pit dug by the male. After spawning, a female takes the eggs and sperm into her mouth, where fertilization takes place, and then leaves the arena (Fryer and Iles 1972; Bruton and Boltt 1975). Parental care is thus restricted to females in this species. There is a marked sexual dimorphism: males are larger than females, have longer anal and dorsal fins, and more robust jaws (Oliveira and Almada 1995). This set of characters seems to play a role in male social behavior such as lateral display, mouth fighting, and mouth digging the spawning pit (Oliveira and Almada 1995).

In this paper we investigate the relationships between gonadal maturation and the expression of behavioral and morphological display characters in O. mossambicus and the possible role of androgens in the coordination of this process.

\section{Methods}

Fish came from a stock bred at our laboratory (for details on this stock see Oliveira 1995). Males ranged from 3.6 to $8.1 \mathrm{~cm}$ standard length, and females ranged from 3.1 to $7.1 \mathrm{~cm}$ standard length. Fish were kept at $24 \pm 20 \mathrm{C}$ with a photoperiod of 12L:12D. Sixteen groups of immature juveniles (ca. 20-30 days post-bucal incubation) of six individuals each were placed in standard aquaria $\left(62 \times 34 \times 39 \mathrm{~cm}^{3}\right)$ and reared until the onset of sexual maturity (ca. 8-9-month-old fish). At this time behavioral observations were carried out. The sex ratio (males:females) in the groups was as follows: one group had 1:5, one group had 2:4, five groups had $3: 3$, seven groups had $4: 2$. Due to mortality during the experiment two groups were reduced to four (1 male: 3 females) and five individuals ( 2 males: 3 females).

After the behavioral observations the individuals were killed with an overdose of the anesthetic quinaldine (Sigma), morpho- 
metric measurements [dorsal fin length (DFL), anal fin length (AFL), caudal fin length (CFL), pre-maxilla width (PW), mandibula width (MW), pre-maxilla length (PL), snout length (SL); for a detailed definition of these measurements see Oliveira and Almada 1995] were taken with a caliper to the nearest $0.1 \mathrm{~mm}$, and the individuals were dissected for gonad inspection. The following measurements were also taken: body weight, gonadal weight, and length of genital papilla (G-PAP) (to the nearest $0.1 \mathrm{~mm}$ using a calibrated micrometer on a stereomicroscope). We included caudal fin length in the present study as a control variable since it does not show any sexual dimorphism (Oliveira 1995). The stage of maturation of the gonads was assessed following a scale for macroscopic inspection proposed by Dadzie (1969) and by Arthingthon and Milton (1986)-gonad maturation index $(1=$ juvenile; 2 = inactive; $3=$ maturing ; $4=$ mature; $5=$ spent - and by using the gonado-somatic index (GSI = gonad weight/body weight $\times 100$ ).

Due to the large number of groups $(n=16)$ we subdivided them into two subsets (of eight groups each) and observed them under different protocols. The first set of groups ( $n=28$ males) was followed for 8 consecutive weeks and observations were carried out following a behavioral sampling procedure with continuous recording (see Martin and Bateson 1993) of agonistic and sexual interactions, in periods of $5 \mathrm{~min}$ each. Each group was observed for six such periods each week, constituting $30 \mathrm{~min}$ of observation per group each week. In these groups, we also recorded the following variables each week: presence of nests and the identity of the nesting male; nest volumes; and the dark coloration intensity for each individual. In the second set of groups $(n=$ 18 males) we used focal animal observations with continuous recording (see Martin and Bateson 1993) of agonistic and sexual interactions. Each group was observed on 2 consecutive days, and on each day six focal observations of 5 min each were performed, summing to $1 \mathrm{~h}$ of observation per fish, and $6 \mathrm{~h}$ per group. In these groups, we also recorded the following variables: presence of nests and the identity of the nesting male, nest volumes, and the dark coloration intensity for each individual. In each group individuals were recognized by natural markings and relative size differences. A detailed description of the agonistic and sexual behavior patterns of $O$. mossambicus may be found in Baerends and Baerends van Roon (1950) and in Neil (1964). The dark coloration intensity was estimated on a scale from 0 to $4(0=$ neutral; $1=$ dark $1 ; 2=$ dark $2 ; 3=$ dark $3 ; 4=$ black) according to the coloration patterns described by Neil (1964). An average coloration intensity was computed for each fish based on 12 measurements of the coloration intensity taken at the beginning of each observation period. Nest volume was estimated from measurements of the diameter and depth at the center of the pits, assuming that the nests have the approximate shape of an inverted cone. To reduce intergroup variability in pit depths each aquarium was provided with approximately the same amount of sand $(7 \mathrm{~cm}$ layer). To estimate the social status of each individual a dominance index (DI) was computed according to the formula: $\mathrm{DI}_{i}=\mathrm{V}_{i} / \mathrm{V}_{i}+\mathrm{D}_{i}$, where $\mathrm{V}_{i}$ is the number of victories and $\mathrm{D}_{i}$ is the number of defeats of each individual $(i)$. This index expresses the success of each fish in the set of agonistic interactions in which it participated (Barlow and Ballin 1976).

As both group sets showed no differences in the trends presented, we merged the compatible data in the analysis. Thus, for the morphological traits, data from both subsets were used. From the 18 males of the second subset, only 8 contributed to this data set $(n=36)$, as 10 of them were used for aromatase assays and morphometric data were not collected. For the behavioral traits, only males of the first subset were used $(n=28)$. Table 1 presents the sample sizes, means, and standard deviations for each variable used.

The length of the genital papilla was used as a measure of androgen levels in males. Levy and Aronson (1955) demonstrated, through castration and exogenous administration of testosterone, that the tilapia genital papilla is an androgen-dependent character and that genital papilla development may be used as a bioassay to evaluate the levels of circulating androgens. This bioassay was validated for $O$. mossambicus by measuring the genital papilla and
Table 1 Descriptive statistics of the variables used in the present study. $D F L$ Dorsal fin length, $A F L$ anal fin length, $C F L$ caudal fin length, $P W$ pre-maxilla width, $M W$ mandibula width, $P L$ pre-maxilla length, $S L$ snout length; $G S I$ gonado-somatic index, GPAP gegnital pupilla length; for more details see text

\begin{tabular}{lcc}
\hline Variables & $n$ & Mean \pm SD \\
\hline Morphological & & \\
DFL (mm) & 36 & $13.4 \pm 4.5$ \\
AFL (mm) & 36 & $12.6 \pm 6.0$ \\
CFL (mm) & 36 & $18.9 \pm 4.4$ \\
PW (mm) & 36 & $2.01 \pm 0.75$ \\
MW (mm) & 36 & $1.21 \pm 0.46$ \\
PL (mm) & 36 & $7.32 \pm 2.39$ \\
SL (mm) & 36 & $8.24 \pm 2.25$ \\
Reproductive state & & \\
GSI $(\%)$ & 36 & $0.68 \pm 0.53$ \\
Maturation (5 scale) & 36 & $3.34 \pm 1.37$ \\
Androgen levels & & \\
GPAP (mm) & 36 & $1.33 \pm 0.70$ \\
Behavioral & & \\
Dominance $(V / V+D)$ & 28 & $0.48 \pm 0.36$ \\
Dark coloration $(4$ scale) & 28 & $2.08 \pm 1.36$ \\
Territory $(\%$ of time) & 28 & $2.78 \pm 5.83$ \\
Courtship rate (acts/5 min) & 28 & $3.6 \pm 7.4$ \\
Nest volume (cm $\left.{ }^{3}\right)$ & 28 & $7.9 \pm 11.5$ \\
Nest digging $(\%$ time) & 28 & \\
Nest hovering $(\%$ time) & 28 & \\
\hline
\end{tabular}

assaying androgen levels (testosterone and 11-ketotestosterone) for 14 adult males. The correlations obtained between these measures confirmed that the genital papilla is a good indicator of androgen concentrations $(r=0.67, P<0.01$; Oliveira and Almada 1998).

Correlation coefficients were used to investigate the relationships between gonadal maturation, the expression of behavioral and morphological characters, and androgen levels as evaluated by genital papilla size in the coordination of this process. When dealing with morphological variables partial correlation coefficients were used to control for the effects of size (i.e. standard length). To control for intergroup variability all variables have been standardized (i.e., standard normal deviates) and the standardized values have been used.

\section{Results}

Table 2 presents the partial correlations between several sexually dimorphic traits in $O$. mossambicus morphology and behavioral reproductive traits, after controlling for the effects of body size (standard length). Caudal fin length was included as a control variable since it was shown not to be sexually dimorphic in this species. Table 2 shows that several of the analyzed morphological traits are positively correlated both with measures of reproductive activity and social dominance. Of these morphological traits, the length of the dorsal and anal fins show the more consistent pattern of correlations with the behavioral variables. It is worth noting that the caudal fin does not present the same pattern of correlations as the dorsal and the anal fins. The displaying characters are thus 
Table 2 Partial correlations between morphological and behavioral variables controlling for body size (standard length); for abbreviations, see Table 1

$* P<0.05 ; * * P<0.01$ $* * * P<0.001$

\begin{tabular}{llllc}
\hline Variable & $\begin{array}{l}\text { Dominance } \\
\text { index } \\
(n=36)\end{array}$ & $\begin{array}{l}\text { Time holding } \\
\text { a territory } \\
(n=28)\end{array}$ & $\begin{array}{l}\text { Spawning pit } \\
\text { volume } \\
(n=28)\end{array}$ & $\begin{array}{l}\text { Courtship rate } \\
(n=28)\end{array}$ \\
\hline DFL & $0.46^{* *}$ & $0.74^{* * *}$ & $0.72^{* *}$ & $0.62^{* *}$ \\
AFL & $0.44^{* *}$ & $0.67^{* *}$ & $0.65^{* *}$ & $0.64 * *$ \\
CFL & 0.27 & 0.15 & 0.12 & 0.23 \\
PW & 0.16 & 0.43 & 0.36 & -0.03 \\
MW & 0.21 & 0.43 & 0.37 & 0.01 \\
PL & $0.33^{*}$ & $0.75^{* * *}$ & $0.66^{* *}$ & 0.22 \\
SL & 0.18 & $0.65^{* *}$ & $0.56^{*}$ & 0.34 \\
\hline
\end{tabular}

Table 3 Partial correlations between morphological variables and gonadal maturation indicators [gonado-somatic index $(G S I)$, gonadal maturation as evaluated by a macroscopic scale, and androgen levels as evaluated by genital papilla size $(G P A P)](n=36)$; for further abbreviations, see Table 1

\begin{tabular}{llll}
\hline Variable & GSI & Maturation & $\begin{array}{l}\text { GPAP } \\
(=\text { androgen levels })\end{array}$ \\
\hline DFL & 0.34 & $0.46^{* *}$ & $0.56^{* * *}$ \\
AFL & 0.37 & $0.52^{* *}$ & $0.61^{* * *}$ \\
CFL & 0.28 & 0.18 & 0.30 \\
PW & 0.29 & $0.47 * *$ & $0.63^{* * *}$ \\
MW & 0.03 & 0.01 & $0.38^{*}$ \\
PL & 0.33 & $0.64 * * *$ & $0.81^{* * *}$ \\
SL & 0.07 & 0.23 & $0.59^{* * *}$ \\
GPAP & $0.45^{* *}$ & $0.64 * *$ & - \\
\hline
\end{tabular}

$* P<0.05 ; * * P<0.01 ; * * * P<0.001$

Table 4 Correlation coefficients (Pearson) between behavioral variables and gonadal maturation indicators

\begin{tabular}{llll}
\hline Variable & GSI & Maturation & $\begin{array}{l}\text { GPAP } \\
(=\text { androgen levels })\end{array}$ \\
\hline Dominance $(n=36)$ & $0.33^{*}$ & $0.39^{*}$ & $0.35^{*}$ \\
Dark color $(n=28)$ & $0.51^{* *}$ & $0.72^{* *}$ & $0.70^{* * *}$ \\
Time holding & 0.38 & $0.73^{* * *}$ & $0.83^{* * *}$ \\
$\quad$ a territory $(n=28)$ & & & \\
Courtship rate $(n=28)$ & 0.15 & $0.50^{*}$ & 0.45 \\
Nest volume $(n=28)$ & 0.32 & $0.72^{* *}$ & $0.77^{* * *}$ \\
Nest digging $(n=28)$ & 0.19 & $0.61^{* *}$ & $0.65^{* * *}$ \\
Nest hovering $(n=28)$ & 0.30 & $0.62^{* *}$ & $0.69^{* * *}$ \\
\hline
\end{tabular}

$* P<0.05 ; * * P<0.01 ; * * * P<0.001$

strongly correlated among themselves and probably share a common underlying causal factor.

The sexually dimorphic characters are best correlated with gonad maturation (DFL, AFL, PW, PL) and androgen levels (DFL, AFL, PW, MW, PL, SL) (see Table 3). The GSI is only significantly correlated with the length of the dorsal and anal fins and with the genital papilla size. Table 3 also shows that maturation and androgen levels are closely related. Thus, dimorphic male traits may reflect both male qualities. It is important to note that apart from being used in this paper as a measure of androgen levels, the white genital papilla of a black territorial male is a very conspicuous trait that may possess a signal value per se. Thus, it was also included in Table 3 as a morphological displaying character.
The behavioral variables involved in territoriality and reproduction are also positively correlated with gonad maturation and androgen levels (see Table 4). The dark coloration and the dominance status are the only variables significantly correlated with GSI. Thus, gonad maturation and androgen levels are strongly reflected in the behavioral and morphological traits considered in this study.

\section{Discussion}

Although a study based on correlations cannot provide definitive evidence on the causal relationships hypothesized in this paper, the evidence presented above is fully compatible with the interpretation that androgens play a mediating role between gonadal maturation and the development of display characters.

It is interesting to note that PL and SL, variables that express mouth size, best correlate with nest volume and time holding a territory. Since in this species the nest is built by mouth digging (Baerends and Baerends van Roon 1950), a larger mouth may enable the male to dig a larger nest quickly. As mentioned above, the jaw morphological complex is probably also important in maintaining a territory due to its role in mouth fighting and frontal display, agonistic behavioral traits strongly involved in territorial disputes (Baerends and Baerends van Roon 1950; Neil 1964; Fryer and Iles 1972). Dorsal and anal fins are in turn involved in lateral display, an important signal in social interactions (Baerends and Baerends van Roon 1950; Neil 1964; Fryer and Iles 1972).

The finding that GSI shows weaker correlations with displaying characters than the level of maturation does not contradict our interpretation. Indeed, fishes with very different GSI may be in the same maturational state and vice versa, as so frequently shown in species with male alternative mating tactics (Taborsky 1994).

The kind of relationship that we propose is only to be expected when males', reproductive success is dependent on their courtship and ability to compete with other males for the access to females, since in sneaker males this coupling between gonadal maturation and the maturation of displaying characters is broken (for a review see Taborsky 1994). It is interesting to note that in species with male sexual polymorphism the two male types 
frequently show different androgen profiles (see Brantley et al. 1993).

Acknowledgements During this study R. Oliveira was supported by a research grant from JNICT (BD/1032/90-IG).

\section{References}

Arthingthon AH, Milton DA (1986) Reproductive biology, growth and age composition of the introduced Oreochromis mossambicus (Cichlidae) in two reservoirs, Brisbane, Australia. Environ Biol Fishes 16:257-266

Baerends GP, Baerends van Roon JM (1950) An introduction to the study of the ethology of cichlid fishes. Behaviour [Suppl] 1: $1-242$

Barlow GW, Ballin PJ (1976) Predicting and assessing dominance from size and coloration in the polychromatic Midas cichlid. Anim Behav 24:793-813

Borg B (1994) Androgens in teleost fishes. Comp Biochem Physiol C Pharmacol Toxicol Endocrinol 109:219-245

Brantley RK, Wingfield JC, Bass AH (1993) Sex steroid levels in Porichthys notatus, a fish with alternative reproductive tactics, and a review of the hormonal bases for male dimorphism among teleost fishes. Horm Behav 27:332-347

Bruton MN, Boltt RE (1975) Aspects of the biology of Tilapia mossambica Peters (Pisces: Cichlidae) in a natural freshwater lake (Lake Sibaya, South Africa). J Fish Biol 7:423-446

Dadzie S (1969) Spermatogenesis and the stages of maturation in the male cichlid fish Tilapia mossambica. J Zool 159:399403

Fostier A, Jalabert RE, Billard R, Breton B, Zohar Y (1983) The gonadal steroids. In: Hoar WS, Randall DJ, Donaldson EM (eds) Fish physiology, vol 9 A. Reproduction - endocrine tis- sues and hormones. Academic Press, New York, pp 277 372

Fryer G, Iles TD (1972) The cichlid fishes of the great lakes of Africa - their biology and evolution. Oliver \& Boyd, Edinburgh

Halliday TR (1987) Physiological constraints on sexual selection. In: Bradbury JW, Andersson MB (eds) Sexual selection: testing the alternatives Wiley, Chichester, pp 247-264

Levy M, Aronson LR (1955) Morphological effects of castration and hormone administration in the male cichlid fish Tilapia macrocephala. Anat Rec 122:450-451

Magnhagen C (1991) Predation risk as a cost of reproduction. Trees 6:183-186

Martin P, Bateson P (1993) Measuring behaviour: an introductory guide. Cambridge University Press, Cambridge, UK

Nagahama Y (1983) The functional morphology of teleost gonads. In: Hoar WS, Randall DJ, Donaldson EM (eds) Fish physiology, vol 9 A. Reproduction - endocrine tissues and hormones. Academic Press, New York, pp 223-275

Neil EH (1964) An analysis of colour changes and social behaviour of Tilapia mossambica. Univ Calif Publ Zool 75:1-58

Oliveira RF (1995) Etologia social e endocrinologia comportamental da tilápia Oreochromis mossambicus (Teleostei, Cichlidae). PhD thesis, University of Lisbon, Lisbon

Oliveira RF, Almada VC (1995) Sexual dimorphism and allometry of external morphology in Oreochromis mossambicus. J Fish Biol 46:1055-1064

Oliveira RF, Almada VC (1998) Androgenization of dominant males in a cichlid fish: androgens mediate the social modulation of sexually dimorphic traits. Ethology 104:841-858

Taborsky M (1994) Sneakers, satellites, and helpers: parasitic and cooperative behavior in fish reproduction. Adv Stud Behav 23:1-100

Communicated by M.E. dos Santos 